\title{
ACTA BORUSSICA
}

Neue Folge

2. Reihe: Preussen als KulturstaAt 


\title{
ACTA BORUSSICA
}

Neue Folge

\section{Reihe: Preussen als KulturstaAt}

Herausgegeben von der

Berlin-Brandenburgischen Akademie der Wissenschaften

\author{
unter der Leitung \\ von
}

Wolfgang Neugebauer 


\title{
Abteilung I \\ Das preußische Kultusministerium als Staatsbehörde und gesellschaftliche

$$
\text { Agentur (1817-1934) }
$$

\author{
Band 1.2
}

Die Behörde und ihr höheres Personal

\section{Dokumente}

Ausgewählt und bearbeitet von

Bärbel Holtz, Rainer Paetau, Christina Rathgeber,

Hartwin Spenkuch, Reinhold Zilch

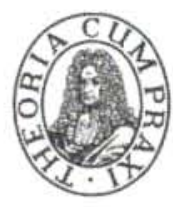

Akademie Verlag 
Dieser Band wurde durch die Gemeinsame Wissenschaftskonferenz im Akademienprogramm mit Mitteln des Bundes (Bundesministerium für Bildung und Forschung) und des Landes Berlin (Senatsverwaltung für Bildung, Wissenschaft und Forschung) gefördert.

Bibliografische Information der Deutschen Nationalbibliothek

Die Deutsche Nationalbibliothek verzeichnet diese Publikation in der Deutschen Nationalbibliografie; detaillierte bibliografische Daten sind im Internet über http://dnb.d-nb.de abrufbar.

ISBN 978-3-05-004572-6

(C) Akademie Verlag GmbH, Berlin 2009

Das eingesetzte Papier ist alterungsbeständig nach DIN/ISO 9706.

Alle Rechte, insbesondere die der Ubersetzung in andere Sprachen, vorbehalten. Kein Teil dieses Buches darf ohne schriftliche Genehmigung des Verlages in irgendeiner Form - durch Photokopie, Mikroverfilmung oder irgendein anderes Verfahren - reproduziert oder in eine von Maschinen, insbesondere von Datenverarbeitungsmaschinen, verwendbare Sprache übertragen oder übersetzt werden.

Einbandgestaltung: Ingo Scheffler, Berlin Lektorat: Gaby Huch, Berlin

Satz: work:at:Book, Martin Eberhardt, Berlin

Druck und Bindung: Druckhaus „Thomas Müntzer“ GmbH, Bad Langensalza

Printed in the Federal Republic of Germany 